\title{
Perspective
}

PERSPECTIVE Actualité en histoire de l'art

1 | 2012

Art et pouvoir

\section{Le souverain en images dans la Sicile normande}

Images of the sovereign in Norman Sicily

Sulamith Brodbeck

\section{(2) OpenEdition}

Journals

Édition électronique

URL : http://journals.openedition.org/perspective/616

DOI : $10.4000 /$ perspective. 616

ISSN : 2269-7721

Éditeur

Institut national d'histoire de l'art

Édition imprimée

Date de publication : 30 juin 2012

Pagination : 167-172

ISSN : 1777-7852

\section{Référence électronique}

Sulamith Brodbeck, "Le souverain en images dans la Sicile normande », Perspective [En ligne], 1 |

2012, mis en ligne le 30 décembre 2013, consulté le 01 octobre 2020. URL : http://

journals.openedition.org/perspective/616; DOI : https://doi.org/10.4000/perspective.616 


\section{Le souverain en images dans la Sicile normande}

\section{Sulamith Brodbeck}

Au cour de la Méditerranée, la Sicile a toujours été un territoire stratégique d'un point de vue à la fois politique et commercial. Au XII ${ }^{\mathrm{e}}$ siècle, sous les Normands, l'île connaît une des périodes les plus florissantes de son histoire, donnant naissance à une civilisation originale marquée par les différentes communautés et par le phénomène d'acculturation ${ }^{1}$. La conquête de la Sicile par les Normands s'achève en 1091 avec la prise de la dernière place forte arabe, la ville de Noto. Elle s'exerce sur une population composite, un microcosme religieux et linguistique sur lesquels les souverains normands greffent leur propre processus d'unification ${ }^{2}$. Hugues Falcand, chroniqueur contemporain de Guillaume II (1166-1189), nous renseigne sur ce procédé : "Le souverain s'informe avec une extrême diligence des coutumes, des rois et des peuples, afin de s'approprier tout ce qu'il pourrait trouver de beau et d'utile ${ }^{3}$. Si cette citation, resituée dans son contexte, s'applique à l'administration royale, elle pourrait tout aussi bien refléter l'attitude des rois normands face à la représentation de leur pouvoir.

Les études récentes, tant en histoire qu'en histoire de l'art, ont mis en avant la symbolique et la mise en scène du pouvoir normand, qui reposait sur la symbiose des grandes cultures du monde méditerranéen par le biais notamment de l'image. Cette dernière fut particulièrement réfléchie, voire instrumentalisée dans les fondations royales qu'ont entreprises les souverains successifs, de Roger II, proclamé roi en 1130, à Guillaume II, mort en 1189 . Véritables rois-bâtisseurs, ils ont commandé la construction d'églises et de cathédrales à Palerme, à Cefalù et à Monreale qui reflètent ce que l'on a souvent appelé le "syncrétisme culturel " de la Sicile normande ; en témoigne l'intérieur de ces édifices, recouvert de mosaïques de tradition "byzantine " sur les parois et de peintures de tradition « islamique " sur les plafonds en bois ${ }^{4}$.

Depuis une quinzaine d'années, ces décors ont fait l'objet d'une nouvelle approche, non plus centrée sur les aspects stylistiques et donc de datation, mais sur la dimension royale de leur programme ${ }^{5}$. Le souverain est mis en images dans les portraits ou panneaux votifs, comme dans le programme iconographique qui l'entoure et le glorifie ; l'image dépasse alors son cadre matériel et renvoie à la représentation que le souverain veut qu'on ait de lui. Ainsi, analyser le " souverain en images " dans ces décors monumentaux nous permet, à travers des études de cas, d'aborder les questions qui animent les recherches actuelles : comment interpréter les éléments byzantins dans l'iconographie du souverain ? Dans quelle mesure peut-on parler de programme iconographique royal ? Enfin, comment les contemporains percevaient-ils ce que les historiens de l'art ont dissocié, à savoir les composantes latine, byzantine et islamique de ces créations?

\section{Les portraits de souverains : Imitatio Byzantii ${ }^{6}$} Les effigies royales conservées dans des décors monumentaux concernent principalement les règnes de Roger II et de Guillaume II $^{7}$. Le plus ancien panneau en mosaïque présente Roger II couronné par le Christ (fig. 1) et se situe dans le narthex de l'église Sainte-Marie-de-l'Amiral à Palerme, appelée plus communément la Martorana, fondée en 1143 par Georges d'Antioche, "émir des émirs " du roi. Les autres panneaux prennent place dans la cathédrale royale de Monreale, érigée sous le règne de Guillaume II, et présentent d'une part le souverain couronné par le Christ trônant (fig. 2) et, d'autre part, le roi offrant le modèle de son église à la Vierge.

Les premiers grands spécialistes des mosaïques siculo-normandes, Otto Demus puis Ernst Kitzinger, se sont intéressés à la composante byzantine de ces portraits, qui mettent en scène le roi normand en costume d'apparat de l'empereur chrétien d'Orient, vêtu d'une longue tunique bleue recouverte du $\operatorname{loros}^{8}$ croisé, richement orné, et portant une couronne ouverte dotée des traditionnelles pendeloques ${ }^{9}$. Ces éminents byzantinistes ont associé ce canon aux modèles iconographiques constantinopolitains de la cour impériale.
1. Roger II couronné par le Christ, vers 1143, Palerme, église Sainte-Marie de l'Amiral. 
2. Guillaume II couronné par le Christ, 11721189, Palerme, cathédrale de Monreale.

3. Ivoire de Constantin VII Porphyrogénète couronné par le Christ, milieu du Xe siècle,

Moscou, Musée Pouchkine.

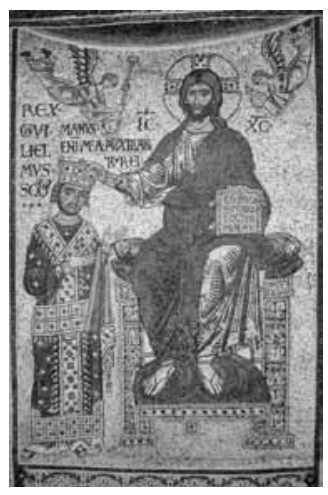

Selon Kitzinger, le panneau de Roger II à la Martorana s'inspire dans les moindres détails d'un modèle iconographique byzantin, tel qu'on peut le voir dans le célèbre ivoire du couronnement de Constantin VII Porphyrogénète du milieu du $\mathrm{X}^{\mathrm{e}}$ siècle, aujourd'hui conservé au musée Pouchkine de Moscou (fig. 3). Seule l'inscription diffère : Roger II est qualifié de "Rhogérios Rêx " et non d'" empereur dans le Christ, Basileus des romains ". À Byzance, le développement $\mathrm{du}$ thème du couronnement symbolique de l'empereur par le Christ ou par une figure déléguée (Vierge, ange ou saint) est favorisé à partir du IX ${ }^{e}$ siècle, à l'époque macédonienne ; il exprime très clairement l'origine divine du pouvoir suprême, fondement de la légitimité du basileus représenté.

Dans la Sicile normande, d'après les recherches sur les sceaux et les monnaies, ou encore l'analyse d'une plaque émaillée conservée dans le trésor de la basilique de Bari, cette iconographie du souverain normand en empereur byzantin est récurrente ${ }^{10}$. Comment interpréter alors la présence en images d'un mode vestimentaire oriental réservé au basileus ? Comme le dit très justement Kitzinger, les recherches sur cette problématique ont souffert d'une orientation trop étroite, les images étant utilisées dans l'unique intention de déterminer la nature des vêtements du roi et des insignes associés à sa personne dans la vie réelle ${ }^{11}$. La critique s'est donc longtemps demandée si le souverain normand portait une telle couronne et un tel vêtement d'origine orientale à la cour palermitaine ${ }^{12}$. Rien dans les sources écrites ou dans les vêtements royaux conservés dans la Weltliche Schatzkammer du Kunsthistorisches Museum de Vienne ne vient cependant appuyer une telle hypothèse ${ }^{13}$. Il est aujourd'hui admis que le souverain normand ne revêtait pas le costume impérial byzantin, comme l'attestent les recherches menées par l'historienne Annliese Nef sur les éléments d'origine islamique dans le costume et le cérémonial royal ${ }^{14}$. La mosaïque de la Martorana ne révèle donc ni la réalité de la cour palermitaine ni celle de la cour constantinopolitaine ${ }^{15}$; elle met en scène les symboles impériaux byzantins comme instrument sémiotique du pouvoir ${ }^{16}$, car ils incarnent l'idéal absolu et universel du pouvoir monarchique que le roi revendique.

Toutefois, une telle représentation signifie-telle pour autant que le roi suit l'idéologie impériale byzantine ? Kitzinger s'est intéressé dès les années 1950 à l'interprétation politique du portrait de Roger II dans l'église de la Martorana ${ }^{17}$, problématique qui fut ensuite principalement traitée par des historiens. Agostino Pertusi, par exemple, s'est demandé dans quelle mesure les pays qui empruntaient à l'art impérial constantinopolitain ne se considéraient-ils pas comme les héritiers en partie ou totalement de la conception politique du pouvoir byzantin ${ }^{18}$. Kitzinger insiste précisément sur le motif de Christomimesis dans le portrait du roi Roger II et affirme que l'image du basileus sert à exprimer non seulement l'ambition de Roger sur le plan international, mais encore le concept byzantin de rex et sacerdos ${ }^{19}$. Une idée similaire du roi Christomimétes est développée par Thomas Dittelbach à propos de Guillaume II dans la cathédrale de Monreale ${ }^{20}$. De manière générale, la critique soutient l'idée d'une volonté idéologique des Normands de se montrer sur un pied d'égalité avec le basileus et Byzance, dont ils cherchaient en vain à conquérir la capitale. Il convient toutefois de s'interroger sur une telle interprétation, comme l'a démontré récemment Mirko Vagnoni dans sa thèse de doctorat consacrée aux représentations

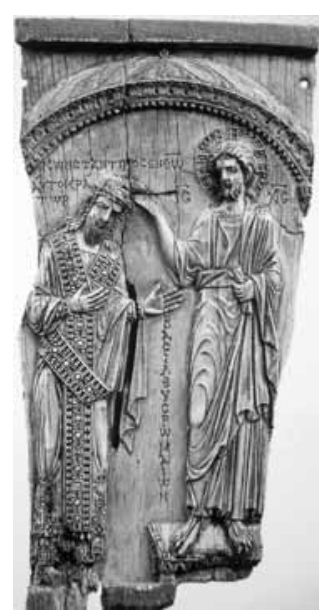
des souverains normands et aux idéologies politiques, dans laquelle il relativise la sacralité divine, absolue, universelle et inconditionnelle des rois normands ${ }^{21}$. Selon lui, l'Imitatio Byzantii reflète avant tout la volonté de la dynastie d'affirmer l'existence d'une autorité unique qui ne dérive de rien ni de personne, sinon 
de Dieu ${ }^{22}$. Les portraits de couronnement, s'ils révèlent de manière évidente le concept du souverain a Deo coronatus, ne signifient pas pour autant que le roi cherchait à récupérer l'idéologie du basileus, lieutenant de Dieu sur terre, icône vivante du Christ, participant à l'omnipotence divine et au gouvernement du monde. D'ailleurs, les inscriptions qui accompagnent le portrait du souverain ne le qualifient jamais d' " empereur dans le Christ " ou d'« imitateur du Christ " mais de « roi ». On empruntait donc à l'iconographie impériale traditionnelle pour ce qu'elle symbolise : un pouvoir absolu, lié à l'idée de stabilité et de continuité - et donc de légitimité - de son détenteur. Cet emprunt est réservé à la mise en images du roi, à l'autolégitimation d'un pouvoir récemment acquis dans un royaume nouvellement unifié.

\section{Programme iconographique royal ?}

Depuis l'ouvrage d'Eve Borsook en 1990, Messages in Mosaic: The Royal Programmes of Norman Sicily ${ }^{23}$, les études se sont essentiellement orientées vers une interprétation royale des décors en mosaïque, que ce soit à travers des analyses générales sur la symbolique du pouvoir dans l'art siculo-normand ${ }^{24}$ ou dans des recherches spécifiques consacrées à un décor et à son organisation par rapport à la place et la figure du roi ${ }^{25}$. Les portraits de souverain, loin d'être des images isolées, s'inscrivent dans un programme iconographique qui leur donne sens. Ainsi, le panneau de Guillaume II couronné par le Christ dans la cathédrale de Monreale s'insère dans un réseau d'images, un tout cohérent et réfléchi qui amplifie sa valeur sémantique. L'iconographie du couronnement à Monreale se distingue de celle de la Martorana par la représentation du Christ assis sur son trône, par le sceptre et le globe portés par des anges, et par l'inscription qui accompagne l'image, extraite du psaume 88 , verset 22 : manus enim mea auxiliabitur ei (fig. 3$)^{26}$. Selon Paolo Delogu, ces mots souligneraient la présence continue du Christ dans les actes du roi ${ }^{27}$.

Avec son étude de 1990, Borsook a été la première à analyser ce panneau en mosaïque dans son contexte programmatique, proposant une vaste interprétation de la Jérusalem céleste au sein de laquelle le roi incarne un nouveau David. Guillaume II, assis sur son trône, se situe sous le panneau votif de son couronnement par le

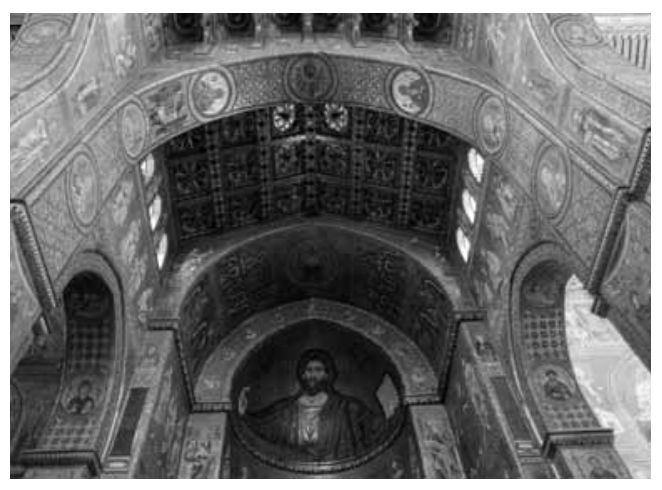

4. Arc surplombant les panneaux votifs, 11721189, Palerme, cathédrale de Monreale.

Christ mais aussi sous la généalogie du Seigneur, comme l'atteste la série des patriarches représentés en médaillons dans l'intrados et au sommet de l'arc (fig. 4). Dans une stricte symétrie, face au panneau de couronnement, se trouve l'image de Guillaume II offrant le modèle de l'église à la Vierge. Le souverain se positionne en tant qu'unique commanditaire et donateur tout en invoquant la protection directe de Marie, titulaire de sa fondation, par une inscription située à l'entrée de l'édifice : « Fiancée de sa descendance (progéniture), étoile qui met au monde le soleil, prie pour tous, mais travaille plus encore pour le roi ".

Toutefois, l'interprétation royale - dans le sens de christologie royale - du programme iconographique atteint parfois ses limites. Certaines des affirmations de Borsook mériteraient d'être nuancées car elles tendent à une surinterprétation du décor. Ainsi, quand l'auteur met en relation l'inscription sur le phylactère du prophète Malachie, situé sur l'arc occidental du chœur, avec le trône de Guillaume II et le cérémoniel de l'acclamation royale, il est primordial de rappeler que ce verset prophétique est avant tout lié à la scène située juste au-dessus, celle de la Présentation de Jésus au grand prêtre Syméon (fig. 5) ${ }^{28}$. Dans un décor comme celui de Monreale, commandé par le roi mais orchestré par de hauts et savants dignitaires ecclésiastiques de la cour, il ne faut pas perdre de vue la complexité des lectures et la polysémie de l'image qui devait rendre compte des multiples fonctions de cette église : une église certes royale, mais tout autant épiscopale et monastique.

Si certains aspects de la christologie royale doivent être nuancés, il n'en demeure pas moins que le programme révèle une véritable dimension politique en raison de la place tenue par le 


\author{
5. Arc occidental \\ du chœur, \\ le prophète \\ Malachie et la \\ Présentation \\ au Temple \\ (gauche), 1172- \\ 1189, Palerme, \\ cathédrale de \\ Monreale.
}

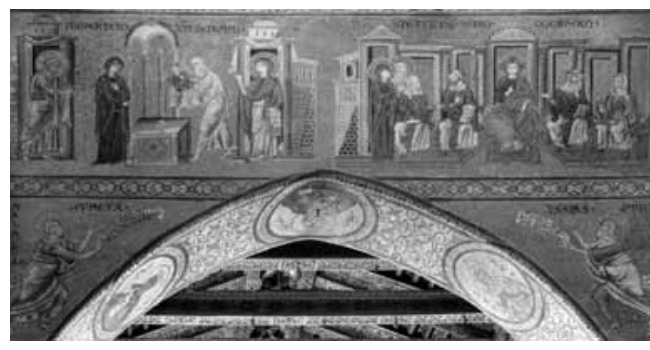

souverain dans cette fondation qui non seulement jouxte le palais de Guillaume II mais est également vouée à devenir mausolée dynastique. Mon étude récente du programme hagiographique, longtemps délaissé par les spécialistes, a révélé dans quelle mesure, plus que toute autre image de l'édifice, les effigies de saints sont particulièrement révélatrices de la politique du souverain et de ses orientations dynastiques $^{29}$. On décèle ainsi la fascination de Guillaume II pour Byzance - à travers l'utilisation des effigies des saints militaires et anargyres orientaux comme protecteurs privilégiés du nouveau souverain et de sa famille -, son alliance par mariage avec le royaume Plantagenêt - visible dans la présence unique en Sicile de Thomas Becket, Hilaire de Poitiers ou Radegonde, situés à des emplacements clés du décor -, et son ancrage dans les traditions locales du Mezzogiorno, attesté par l'importance donnée aux saints régionaux ${ }^{30}$. Ce réseau d'effigies, lié au mobilier cérémoniel et liturgique, qu'il s'agisse du trône du souverain ou des tombes royales, entoure Guillaume II et délimite un espace qui lui est réservé dans la partie orientale de l'église. Il met en avant l'image du roi non plus dans sa dimension portraitiste mais dans une vaste allégorie politique, une des fonctions principales de ces images de saints étant d'exprimer la mission et le rôle du roi à l'échelle de la chrétienté comme à l'échelle locale. Est ainsi mise en exergue la représentation que le pouvoir veut qu'on ait de lui dans un royaume au caractère composite, qui a peu de consistance si ce n'est celle du roi qui le gouverne.

\section{Peut-on encore parler de " composante byzantine " ?}

Dans les études actuelles sur les images du souverain, et plus largement sur l'art et le pouvoir dans la Sicile normande, il demeure un aspect qui mériterait d'être approfondi, celui de la réception de ces images. La composante byzantine des portraits royaux, et plus largement la mosaïque, considérée comme l'art et la technique de Byzance par excellence, étaient-elles perçues par les contemporains comme une "influence " venue d'ailleurs, un élément " étranger " ? Il est intéressant de constater que les descriptions médiévales de portraits aujourd'hui disparus ne se donnent pas la peine de distinguer le costume "byzantin " ou " oriental " du roi, ou encore de faire valoir la mosaïque. C'est notamment le cas des panneaux royaux disparus de la façade de Cefalù, connus grâce à une compilation de documents anciens faite en $1329^{31}$. Ces descriptions parlent d'images sans en préciser la technique et mentionnent le "vêtement royal " et la « couronne " sans insister sur son origine orientale. L'exemple du plafond à muqarnas de tradition islamique de la Chapelle Palatine, reconsidéré récemment par Annliese Nef, est également éclairant à cet égard ${ }^{32}$. Il met en scène des animaux, des motifs végétaux et des figures, dites " profanes " dont des représentations de souverains -, se livrant à différents divertissements. Dans le leitmotiv du " syncrétisme culturel " de la Sicile normande, ce décor a souvent été interprété comme le reflet de la volonté de Roger II d'intégrer les composantes culturelles de la population arabo-musulmane au mode de représentation du pouvoir ${ }^{33}$. Toutefois, dans la célèbre Ekphrasis de Philagète de Cerami, actif à la cour de Roger II, rien ne fait référence à la tradition islamique ; l'auteur décrit le plafond peint en ces mots : une "imitation des cieux transparents du Paradis, illuminés d'un cœur d'étoiles ${ }^{34}$. Ces images ne revendiqueraient donc pas un attachement à une tradition précise, que celle-ci soit byzantine, latine ou arabe. Émettre une telle hypothèse nécessite d'aller au-delà du poids de l'historiographie qui, dans le domaine artistique, témoigne d'un cloisonnement des études : les mosaïques sont longtemps restées la chasse gardée des historiens de l'art byzantin, tout comme le plafond de la Chapelle Palatine celle des spécialistes de l'Islam.

Or, si ces approches ont été utiles et nécessaires, il semblerait qu'il faille reconsidérer cette notion de " composantes ". Ces images reflètent avant tout la spécificité siculo-normande créée par une nouvelle dynastie qui aurait puisé dans une koinè du monde méditerranéen afin de faire valoir l'universalité de sa royauté. Cette dimension 
universelle est illustrée aussi bien dans les images du souverain que dans l'usage des différentes langues sur les monnaies. Le tari d'or sous Roger II est frappé, au droit, de la titulature royale en arabe qui le désigne " puissant par la grâce de Dieu " (al-mu'tazz bi-llāh) et "vénérable " (almu'azzam) et, au revers, d'une croix qui divise le champ en quatre parties portant l'inscription en langue grecque "Jésus Christ vainc " (IC XC NI $\mathrm{KA})^{35}$. Ainsi, les images reflètent l'idéologie du pouvoir des rois normands qui ne répond à aucun canon spécifique. Comme l'a avancé l'historien Jean-Marie Martin : si le souverain « n'est vraiment ni émir, ni basileus, ni roi 'féodal' dans la tradition post-carolingienne, il est de tout cela un peu, sans que cette singularité permette de donner, de lui-même, une image unique ${ }^{36}$.

1. "Le caractère de carrefour culturel de la région n'est pas un mythe ", affirme Jean-Marie Martin dans Italies Normandes, $X I^{e}$-XII $I^{e}$ siècles, Paris, 1994, p. 83. Sur les problèmes d'acculturation, voir les pages 79-129 de ce même ouvrage.

2. Voir Francesco Giunta, "Sicile, Siciliens, sicilitude ", dans Henri Bresc, Geneviève Bresc-Bautier éd., Palerme 10701492. Mosaïque de peuples, nation rebelle : la naissance violente de l'identité sicilienne, Paris, 1993, p. 23-31, ici p. 27.

3. Hugues Falcand, La Historia o Liber de Regno Siciliae e la Epistola ad Petrum panormitane ecclesie thesaurarium, G. B. Siragusa éd., (Fonti per la Storia d'Italia, XXII), Rome, 1897.

4. Le plafond peint le plus célèbre est bien entendu celui de la Chapelle Palatine, toutefois les cathédrales de Cefalù et de Monreale développaient également un décor similaire ; voir Maria Giulia Aurigemma, Il cielo stellato di Ruggero II: il soffitto dipinto della cattedrale di Cefalù, Milan, 2004.

5. Voir infra n. 23, 24 et 25.

6. Cette expression est empruntée à Mirko Vagnoni, "Problemi di legittimazione regia : Imitatio Byzantii" , dans Eduardo D'Angelo, Claudio Leonardi éd., Il Papato e i Normanni: temporale e spirituale in età normanna, (colloque, Ariano Irpino, 2007), Florence, 2011, p. 175-190. Je remercie ici vivement l'auteur de m'avoir envoyé les épreuves de ses travaux.

7. Un seul portrait de Guillaume $\mathrm{I}^{\mathrm{er}}$ est connu par une source médiévale qui décrit les panneaux, aujourd'hui perdus, de la cathédrale de Cefalù : Corrado Mirto éd., Rollus rubeus, Privilegia ecclesie Cephaleditane, a diversis regibus et imperatoribus concessa, recollecta et in hoc volumine scripta, (Documenti per servire alla storia di Sicila, série 1, vol. 29), Palerme, 1972. Voir Mark J. Johnson, " The Lost Royal Portraits of Gerace and Cefalù Cathedrals ", dans Dumbarton Oaks Papers, 53, 1999, p. 237-262.
8. Attribut du costume impérial, désignant une large écharpe brodée ou ornée de gemmes et de perles, enroulée autour du corps et dont un pan retombe sur le bras gauche.

9. Otto Demus et Ernst Kitzinger consacrent une partie de leurs ouvrages à l'étude des « Dedicatory images " ou " Dedication panels ", dans Otto Demus, The Mosaics of Norman Sicily, (Londres, 1949) New York, 1988, p. 302-304 ; Ernst Kitzinger, " On the Portrait of Roger II in the Martorana in Palermo ", dans Studies in Late Antique Byzantine and Medieval Western Art, II, Londres, 2003, p. 1055-1064 [éd. orig. : Proporzioni: studi di storia dell'arte, 3, 1950, p. 30-35] ; Ernst Kitzinger, The Mosaics of St Mary of the Admiral in Palermo, (Dumbarton Oaks Studies, 27), Washington, 1990, p. 189-197.

10. Sur les sceaux et les monnaies, voir Arthur Engel, Recherches sur la numismatique et la sigillographie des Normands de Sicile et d'Italie, Paris, 1882 ; Lucia Travaini, La monetazione nell'Italia normanna, Rome, 1995 ; Sigfrid H. Steinberg, "I ritratti dei re normanni di Sicilia ", dans La Bibliofilia, 39, 1937, p. 29-57 ; pour la plaque émaillée représentant Roger II couronné par saint Nicolas, se référer à Nobiles Officinae: perle, filigrane e trame di seta dal Palazzo Reale di Palermo, Maria Andaloro éd., (cat. expo., Palerme, Palazzo dei Normanni/ Vienne, Hofburg, Schweizerhof, Alte Geistliche Schatzkammer, 2003-2004), I, 2006, p. 216-217.

11. Kitzinger, 1990, cité n. 9, p. 191-192.

12. Hubert Houben déclare : "We do not know whether Roger ever in fact wore such a crown and dress " (Hubert Houben, Roger II of Sicily: A ruler Between East and West, Cambridge/New York, [1997] 2002, p. 115 et n. 35) ; voir également Kitzinger, 1990, cité n. 9, p. 192 et n. 349.

13. En ce qui concerne les sources écrites, se référer aux Ordines pour le couronnement des rois normands : Reinhard Elze, " Tre ordines per l'incoronazione di un re e di una regina del regno normanno di Sicila ", dans Atti del Congresso internazionale sulla Sicilia normanna, (colloque, Palerme, 1972), Palerme, 1973, p. 438-459; pour les vêtements luxueux réalisés dans les ateliers royaux palermitains et aujourd'hui conservés à Vienne, dont le célèbre manteau de Roger II, se référer au catalogue d'exposition Nobiles Officinae..., 2006, cité n. 10.

14. Annliese Nef, « La sphère de la souveraineté. Les images du roi ", dans Annliese Nef, Conquérir et gouverner la Sicile islamique aux XI et XII siècles, (BEFAR, 346), Rome, 2011, p. 119-176, et plus particulièrement la partie dédiée aux " nouveaux éléments concernant le cérémonial royal : insignes, costumes et processions ", p. 121-136.

15. Dans les mosaïques siciliennes, aussi bien la forme de la couronne que celle du loros ne sont plus d'actualité à Byzance pendant la seconde moitié du XII ${ }^{\mathrm{e}}$ siècle. Depuis le $\mathrm{XI}^{\mathrm{e}}$ siècle, la plupart des portraits d'empereurs byzantins présentent en effet le port du loros " modifié " ou "simplifié ", voir Kitzinger, 1990, cité n. 9, p. 192.

16. Se référer à l'étude de Salvatore Tramontana, Vestirsi e travestirsi in Sicilia: abbigliamento, feste e spettacoli nel Medioevo, Palerme, 1993. L'auteur insiste notamment sur l'habit comme message sémiotique précis et opérationnel, comme représentation visuelle et spectaculaire du pouvoir.

17. L'auteur parle de "political manifesto ", dans Kitzinger, 2003, cité n. 9. 
18. Agostino Pertusi, "Insegne del potere sovrano e delegato a Bisanzio e nei paesi di influenza bizantina ", dans Simboli e simbologia nell'Alto Medioevo, (Settimane di Studio, XXIII, 1-2), II, Spolète, 1976, p. 481-563, en particulier p. 561. Voir également les monographies sur Roger II et Guillaume II : Houben, (1997) 2002, cité n. 12, p. 113-135 ( Images of Rule ") ; Annkristin Schlichte, Der "gute " König: Wilhelm II. von Sizilien (1166-1189), (Bibliothek des Deutschen Historischen Instituts in Rom, 110), Tübingen, 2005, p. 223-232 ( "Die Herrschaftsrepräsentation »).

19. Kitzinger, 2003, cité n. 9, p. 1057 ; Kitzinger, 1990, cité n. 9, p. 196. L'idée de Christomimétes est reprise dans Houben, (1997) 2002, cité n. 12, p. 113. Pour la notion de rex et sacerdos, voir Gilbert Dagron, Empereur et prêtre : étude sur le " césaropapisme " byzantin, Paris, 1996, et l'analyse de ce livre faite par Évelyne Patlagean, "Byzance et la question du roi-prêtre ", dans Annales, Histoire, Sciences Sociales, 55/4, 2000, p. 871-878.

20. L'étude est toutefois élargie à l'ensemble du programme dit " royal " et n'est plus limitée uniquement au portrait du souverain ; voir Thomas Dittelbach, « Der Dom in Monreale als Krönungskirche. Kunst und Zeremoniell des 12. Jahrhunderts in Sizilien ", dans Zeitschrift für Kunstgeschichte, 62, 1999, p. 464493, en particulier p. 485.

21. Mirko Vagnoni, Raffigurazioni regie ed ideologie politiche: i sovrani di Sicilia dal 1130 al 1343, thèse, Università degli Studi di Firenze, 2008 ; Mirko Vagnoni, La sacralità regia dei Normanni di Sicilia: un mito?, à paraître ; Vagnoni, 2011, cité n. 6 ; Mirko Vagnoni, "Rex et sacerdos e christomimetes. Alcune considerazioni sulla sacralità dei re normanni di Sicilia ", dans Archivio Normanno-Svevo: rivista di studi sul mondo euromediterraneo dei secoli XI-XIII del Centro Europeo di Studi Normanni, 3, 2012.

22. Comme le rappelle Salvatore Fodale, le roi prétend être le légat permanent et unique du pape dans l'île et s'estime en droit d'intervenir très largement dans le domaine religieux, s'occupant personnellement de la politique ecclésiale de la Sicile : Salvatore Fodale, Comes et legatus Siciliae: sul privilegio di Urbano II e la pretesa Apostolica Legazia dei Normanni di Sicilia, (Università di Palermo, Istituto di Storia medioevale. Studi, 2), Palerme, 1970, et L'Apostolica Legazia e altri studi su Stato e Chiesa, (Historica, 5) Messine, 1991.

23. Eve Borsook, Messages in Mosaic: The Royal Programmes of Norman Sicily, Oxford, 1990.

24. Voir, par exemple : Beat Brenk, "La symbolique du pouvoir ", dans Les Normands, peuple d'Europe, 1030-1200, Mario D’Onofrio éd., (cat. expo., Rome, Palazzo Venezia, 1994), Paris, 1994, p. 179-184 ; Hjalmar Torp, " Politica, ideologia e arte intorno a re Ruggero II ", dans Medioevo: immagini e ideologie, (colloque, Parme, 2002), Milan, 2005, p. 448-458 ; Pina Belli d'Elia, "Liturgie del potere: i segni visivo-oggettuali ", dans Nascita di un regno: poteri signorili, istituzioni feudali e strutture sociali nel Mezzogiorno normanno (1130-1194), (colloque, Bari, 2006), Bari, 2008, p. 367-394.

25. Pour la Chapelle Palatine, voir William Tronzo, The Cultures of His Kingdom, Roger II and the Cappella Palatina in Palermo, Princeton, 1997, et les ouvrages récents qui consacrent des chapitres à l'interprétation palatine et royale de la chapelle : Beat Brenk éd., La Cappella Palatina a Palermo, 4 vol., (Mirabilia Italiae, 15), Modène, 2010 ; Thomas Dittelbach éd., Die Cappella Palatina in Palermo: Geschichte, Kunst, Funktionen, Künzelsau,
2011. Pour la cathédrale de Cefalù, voir Mark J. Johnson, "The Episcopal and Royal Views at Cefalù ", dans Gesta, 33/2, 1994, p. 118-131. Pour la cathédrale de Monreale, voir Thomas Dittelbach, Rex Imago Christi: der Dom von Monreale, Bildsprachen und Zeremoniell in Mosaikkunst und Architektur, (Spätantike, frühes Christentum, Byzanz, 12), Wiesbaden, 2003 ; Thomas Dittelbach, " The Image of the Private and the Public King in Norman Sicily ", dans Römisches Jahrbuch der Bibliotheca Hertziana, 35 , 2003-2004, p. 149-172 ; Rosa Bacile, " Stimulating Perceptions of Kingship: Royal Imagery in the Cathedral of Monreale and in the Church of Santa Maria dell'Ammiraglio in Palermo ", dans Al-Masāq, 16/1, 2004, p. 17-52 ; Sulamith Brodbeck, Les Saints de la cathédrale de Monreale: iconographie, hagiographie et pouvoir royal en Sicile à la fin du XII siècle, (Collection de l'École française de Rome, 432), Rome, 2010.

26. Voici l'intégralité des versets 21 et 22 du psaume 88 : " J'ai trouvé David mon serviteur, je l'ai oint de mon huile sainte ; pour lui ma main sera ferme, mon bras aussi le rendra fort ".

27. Paolo Delogu, "Idee sulla regalità : l'eredità normanna ", dans Potere, società e popolo tra età normanna ed età sveva (11891210), (colloque, Bari/Conversano, 1981), (Centro di studi normanno-svevi. Università degli studi di Bari. Atti, 5), Bari, 1983, p. 185-214, en particulier p. 204.

28. Le texte choisi, "Voici que je vais envoyer mon messager (pour qu'il fraye un chemin devant moi) " (Malachie 3:1), figure dans la liturgie de la fête de la Présentation et illustre directement l'entrée triomphale du Seigneur dans son temple.

\section{Brodbeck, 2010, cité n. 25.}

30. Les détails de cette interprétation sont donnés dans Brodbeck, 2010, cité n. 25, p. 85-115 et p. 145-169.

31. Voir supra n. 7.

32. Nef, 2011, cité n. 14, p. 145-174.

33. Voir notamment Jeremy Johns, "I re normanni e i califfi fatimidi. Nuove prospettive su vecchi materiali ", dans Biancamaria Scarcia Amoretti éd., Del nuovo sulla Sicilia musulmana, (colloque, Rome, 1993), Rome, 1995, p. 9-50, en particulier p. 25.

34. Maria Luigia Fobelli, « L'ekphrasis di Filagato da Cerami sulla Cappella Palatina e il suo modello ", dans Arturo Carlo Quintavalle éd., Medioevo: $i$ modelli, (colloque, Parme, 1999), Milan, 2002, p. 267-275 ; Anneliese Nef précise à propos de la description de Philagète de Cerami : " On voit donc que cette lecture contemporaine du plafond, la seule disponible, banalise ce qui nous paraît si exceptionnel et montre qu'il était sans doute perçu comme une décoration parmi d'autres, même s'il était particulièrement imposant" (Nef, 2011, cité n. 14, p. 162).

35. Houben, (1997) 2002, cité n. 12, p. 121.

36. Martin, 1994, cité n. 1, p. 260.

Sulamith Brodbeck, Université Paris 1 Panthéon-Sorbonne Sulamith.brodbeck@univ-paris1.fr

Mots-clés
Basileus, Byzance, Mosaïque, Normands, Sicile

